\title{
EXPONENTIALLY IMPROVED STATIONARY PHASE APPROXIMATIONS FOR DOUBLE INTEGRALS
}

\author{
D. Kaminski
}

\begin{abstract}
It is well-known that the accuracy of asymptotic expansions can be improved for even moderate values of the asymptotic parameter if one includes exponentially small terms in the expansion. However, in most incarnations of the two-dimensional stationary phase method, this is never done, even though the practice of including subdominant terms is commonplace for the asymptotic analysis of one-dimensional oscillatory integrals with a large parameter.

In this paper, it is shown how one may meaningfully determine appropriate exponentially small terms for inclusion in the asymptotic expansion of an oscillatory double integral over a domain in the real plane. The approach involves the use of surfaces of steepest descent through complex saddle points, and is detailed for an oscillatory integral with no real critical points.
\end{abstract}

\section{Introduction}

In Professor F. W. J. Olver's monograph, Asymptotics and Special Functions, it is pointed out that numerically useful asymptotic expansions can result when one includes exponentially small terms in the expansion. Professor Olver illustrates this with the example of the expansion of

$$
S(m)=\frac{2}{\pi} \int_{0}^{\infty}\left(\frac{\sin t}{t}\right)^{m} d t
$$

evaluated at $m=4$ [Olv, Ch. $3, \S 10.3]$. He further remarks that this observation had been made far earlier, as far back as Stokes [Olv, p. 105].

The inclusion of exponentially subdominant terms is now common practice in precision asymptotics, but does not appear to have played much of a rôle in the asymptotic analysis of oscillatory multidimensional integrals. The usual approach there, for twodimensional integrals, is to observe that if the smooth phase function $f$ in a double integral

$$
I(\lambda)=\iint_{D} g(x, y) e^{i \lambda f(x, y)} d x d y
$$

has no critical point in $D$, and if the amplitude $g$ is smooth throughout $D$, then the principal asymptotic behaviour of (1.1) comes from points where $\partial D$ has a discontinuously turning tangent, or where the level set $f=$ constant is tangent to $\partial D$. Standard techniques (based on partial integration using Green's formula) result in an asymptotic

Received September 24, 1992, revised June 17, 1993.

1991 Mathematics Subject Classification. Primary 41A60, secondary 30E15, 32A30.

Key words and phrases. Asymptotic expansion, two-dimensional oscillatory integral, saddle point, method of steepest descent, surface of steepest descent.

This work was supported, in part, by an NSERC operating grant, and a grant from the University of Lethbridge Research Fund. 
expansion of $I(\lambda)$ for large $\lambda$ expressed solely in terms of these "boundary terms." In particular, if $g$ is compactly supported in the interior of $D$, stationary phase yields the singularly unsatisfactory result: $I(\lambda)=\mathcal{O}\left(\lambda^{-n}\right)$ for all $n=0,1,2, \ldots$; see, for example, the monographs [Jones], [Wong] or [BH].

However, if the amplitude and phase functions appearing in (1.1) are analytic in each of their arguments in a domain in $\mathbb{C} \times \mathbb{C}$, then something more can be said. In this setting, M. V. Fedoryuk showed that one can modify the saddle point method so as to allow the use of complex saddle points in the development of the asymptotics of $I(\lambda)$. One proceeds by deforming the domain $D$ into a new domain $D^{\prime}$ in $\mathbb{C} \times \mathbb{C}$ for which $\partial D=\partial D^{\prime}$, and for which Re if decreases along this deformation. The idea, then, is to replace (1.1) by a similar expression, but now integrated over the new domain $D^{\prime}$. In this way, one may bring complex saddle points into the large $\lambda$ analysis of the behaviour of $I(\lambda)$; see [Fed] (or [Kam1] for an English language summary of these ideas). Two difficulties with this approach, however, are the deformation process used to arrive at the new integration domain $D^{\prime}$, and some measure of ambiguity in choosing branches of surds that appear in the computation of the contribution of the complex saddle point.

Theoretical work [Pham] that replaces the domain $D=\mathbb{R}^{2}$ (for the case of double integrals) by a sum of "Lefschetz thimbles" more closely parallels the operation of the steepest descent method, in the sense that an integration "contour" $D$ without boundary is replaced by a sum of integration cycles, each of which contains a critical point of the phase, and over which the function if has decreasing real part (as one moves away from the critical point), and constant imaginary part. Unfortunately, the work in [Pham] is of an abstract character, and gives no indication as to how one can construct, in a practical fashion, the equivalent integration cycle(s).

A more constructive approach has been taken by F. Ursell, in which the asymptotics of a function defined by a double integral in $\mathbb{C} \times \mathbb{C}$ are developed; see [Urs]. In this setting, the surface over which the integral is defined is constructed with the use of steepest descent curves. This construction is mentioned again in the final section of this paper.

Practical use has also been made of the contributions made to oscillatory integrals by complex saddle points. In [IN], Ikuno and Nishimoto observe that a stationary phase approximation of a double integral in an optics problem can be made to yield numerically improved values if certain complex stationary points are allowed into the asymptotic approximation. One goal of this paper is to set some of their work into a more rigorous framework, showing, in particular, which of the complex stationary points should be included in the development of an asymptotic approximation.

It should be noted that the contributions of complex saddle points to the asymptotic behaviour of oscillatory integrals can be expected to play a significant rôle in the study of hyperasymptotics of multidimensional integrals; see [BeH, p. 673].

\section{A special case}

As is often the case with asymptotics, we will not be presenting and proving a suite of general theorems. Rather, we will select one reasonably representative example, and illustrate our approach to the problem by carefully examining this example in some detail. With that restricted aim, we will establish the following result: 
Theorem 2.1. Let $I(\lambda)$ be the function defined by

$$
I(\lambda)=\iint_{D} e^{\lambda S(z, w)} d z d w
$$

where

$$
S(z, w)=i\left(w^{2}+1\right)\left(z^{3} / 3+z\right)
$$

and $D$ is a real, bounded domain in the real $(z, w)$-plane. If $D$ contains the origin, then the asymptotic expansion of (2.1), as $\lambda \rightarrow+\infty$, includes a contribution of the complex saddle $(i, 0)$ of the form

$$
\frac{\pi}{\lambda} \sqrt{\frac{3}{2}} e^{-2 \lambda / 3} \sum_{j=0}^{\infty} a_{j} \lambda^{-j / 2}
$$

where $a_{0}=1$.

We will later wish to compare numerical values for $I(\lambda)$ with values obtained from asymptotic estimates. For that comparison, we will use an integration domain of $[-1,1] \times[-1,1]$. This domain will exhibit two types of boundary critical points: corners, and points of tangency of the boundary with level sets of $\operatorname{Im} S$.

Although the integral $I(\lambda)$ in (2.1) is a simple one, this will serve as an adequate basis for the analysis of more general integrals, for a number of reasons. First, many analytic functions can be represented in a canonical form as a polynomial - thus, in some cases, one need only consider polynomial phase functions $S$. Second, the choice of a constant amplitude function is not overly restrictive, as a more general class of analytic amplitudes does not introduce any new difficulties - we would only need to conduct a termwise integration of the amplitude's Taylor series centered at the critical point in order to accommodate non-constant amplitudes. Finally, for non-polynomial phases, one would need to make greater use of numerical solutions of autonomous systems.

Observe that the phase function $(2.2)$ has a total of eight critical points: $( \pm i, 0)$, $(0, \pm i)$, and $( \pm i \sqrt{3}, \pm i)$. We note that $S(i, 0)=-2 / 3, S(-i, 0)=2 / 3$ and $S$ (any other critical point) $=0$, so that, in every case, $\operatorname{Im} S=0$ at a critical point. We shall determine which critical points are relevant to the asymptotic behaviour of (2.1) by employing Fedoryuk's deformation process.

\section{The deformation process in the plane}

Prior to proceeding with the analysis of the double integral (2.1), it is perhaps helpful to first examine the technique we shall be using in the more familiar setting of the complex plane. Let

$$
J(\lambda)=\int_{-1}^{1} e^{i \lambda\left(x^{3} / 3+x\right)} d x
$$

where $\lambda$ is, as before, a large positive parameter. It is easy to show [Wong, Chap. 1, $\S 2]$ that $J$ has the asymptotic behaviour

$$
J(\lambda)=\frac{1}{\lambda} \sin \left(\frac{4 \lambda}{3}\right)-\frac{1}{2 \lambda^{2}} \cos \left(\frac{4 \lambda}{3}\right)+\mathcal{O}\left(\frac{1}{\lambda^{3}}\right), \quad \lambda \rightarrow+\infty .
$$


We shall show that, by appropriately deforming the initial integration contour, the closed interval $[-1,+1]$, we can also include an exponentially negligible term.

The approach we take to accomplish this begins by constructing all trajectories of the gradient system

$$
(\dot{u}, \dot{v})=-\nabla \operatorname{Re} i\left((u+i v)^{3} / 3+(u+i v)\right)=\left(2 u v, u^{2}-v^{2}+1\right)
$$

beginning at points of the real interval $[-1,+1]$, i.e., satisfying the initial conditions $u(0)=u_{0}, v(0)=0$, for $u_{0} \in[-1,+1]$. Here, we make the identification $x=u+i v$

We note that if $u$ ever vanishes, then $\dot{u}=0$ and $\dot{v}=1-v^{2}$. Thus, the trajectory issuing from $u=v=0$ never leaves the $v$-axis, and further, can never pass through the point where $v=1$, which gives an equilibrium point for the system. Since the initial conditions for each trajectory have $v(0)=0$, every trajectory moves off the $u$-axis into the upper half plane.

The union of the system's trajectories produces a planar region bounded by the interval $[-1,+1]$, two curves issuing from \pm 1 into the upper half plane $v>0$, and a curve which passes through the point $x=i$; see Fig. 1 .

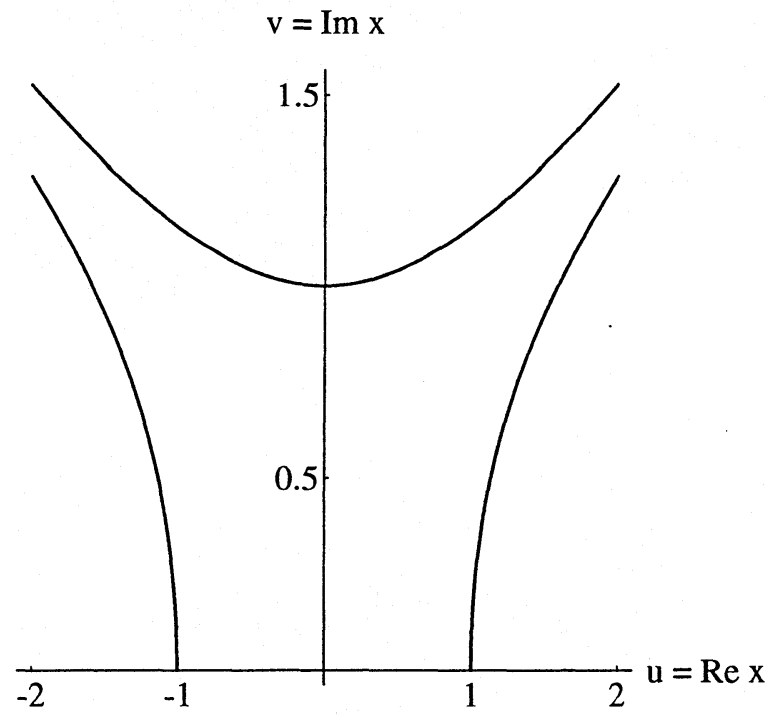

Figure 1. The planar region generated by trajectories issuing from $[-1,+1]$.

As is known from classical steepest descent methodology, every trajectory constructed with this autonomous system is part of a level curve $\operatorname{Im} i\left(x^{3} / 3+x\right)=$ const. In particular, the trajectory through $\left(u_{0}, 0\right)$ in the $u v$-plane passes along a component of

$$
\frac{1}{3} u^{3}-u v^{2}+u=\frac{1}{3} u_{0}^{3}+u_{0}
$$

In the case of $u_{0}=0$, this simplifies to the curves $u=0$ and $u^{2} / 3-v^{2}+1=0$. This last possibility yields the bounding curve of the planar region which passes through the point $x=i$. 
Because of the exponential decay in the integrand of $J$, we may write

$$
J(\lambda)=-\int_{1}^{\infty e^{\pi i / 6}} e^{i \lambda\left(x^{3} / 3+x\right)} d x+\int_{-1}^{\infty e^{5 \pi i / 6}} e^{i \lambda\left(x^{3} / 3+x\right)} d x+\int_{\infty e^{5 \pi i / 6}}^{\infty e^{\pi i / 6}} e^{i \lambda\left(x^{3} / 3+x\right)} d x
$$

the various contours for these integrals are the bounding arcs of the region containing the trajectories issuing from $[-1,+1]$, and are illustrated in Fig. 1. Routine computation reveals that the last integral in (3.3) is expressible as $2 \pi \cdot \lambda^{-1 / 3} \cdot \operatorname{Ai}\left(\lambda^{2 / 3}\right)$; the remaining two integrals in (3.3) give rise to the usual asymptotic expansion one obtains for $J(\lambda)$, given earlier as (3.2).

With this Airy function term included, our asymptotic approximation can be improved to

$$
J(\lambda)=\frac{1}{\lambda} \sin \left(\frac{4 \lambda}{3}\right)-\frac{1}{2 \lambda^{2}} \cos \left(\frac{4 \lambda}{3}\right)+\frac{2 \pi}{\lambda^{1 / 3}} \cdot \operatorname{Ai}\left(\lambda^{2 / 3}\right)+\mathcal{O}\left(\frac{1}{\lambda^{3}}\right), \quad \lambda \rightarrow+\infty .
$$

That the Airy function term is indeed of exponentially small order is readily apparent from its well-known asymptotic expansion.

The inclusion of an asymptotically negligible term such as the Airy function term can render the resulting approximation far more useful numerically at modest values of the argument $\lambda$. In the present case, for $\lambda$ as small as 2 , the improved approximation commits a relative error of $7 \%$, whereas the simpler expression at $\lambda=2$ produces a relative error of $45 \%$.

\section{Constructing the steepest descent surface}

Our starting point in the construction of the steepest descent surface(s) (the integration chains of Pham) is the autonomous system used by Fedoryuk to perform the deformation of the original integration domain $D$. The process mimicks closely the planar situation in the previous section.

Since we wish to replace $D$ by a new two-dimensional domain over which $\operatorname{Im} S$ is constant, and over which max $\operatorname{Re} S$ is achieved only at the critical point, we consider the curves of steepest descent of $S$ issuing from points of $D$, viz.,

$$
(\dot{x}, \dot{y}, \dot{u}, \dot{v})=-\nabla \operatorname{Re} S(x, y, u, v),
$$

where we have identified $z$ with $x+i y, w$ with $u+i v, x, y, u$, and $v$ all real, and we write $S(z, w) \equiv S(x, y, u, v)$. Here, the gradient is taken with respect to $x, y, u$ and $v$. The parameter $t$ is a nonnegative real number, so that $(x(0), y(0), u(0), v(0))$ is always a point of $D$, and $\operatorname{Re} S(x(t), y(t), u(t), v(t))$ decreases with increasing $t$. It is easy to show that along a trajectory of (4.1), we have $\operatorname{Im} S=\operatorname{Im} S(x(0), y(0), u(0), v(0))$.

Note that we cannot define a steepest descent surface merely by setting $\operatorname{Im} S=$ $\operatorname{Im} S\left(z_{0}, w_{0}\right)$, as is the case for steepest descent in the plane, since such a level hypersurface has dimension three - one dimension too many for our integral. Note, too, that a saddle point $\left(z_{0}, w_{0}\right)$ of $S$ is a singular point of the variety $\operatorname{Im} S=\operatorname{Im} S\left(z_{0}, w_{0}\right)$.

Let us call a saddle point $\left(z_{0}, w_{0}\right)$ accessible from the domain $D$ if there is a trajectory of (4.1) issuing from a point of $D$ such that

$$
\left(z_{0}, w_{0}\right)=\lim _{t \rightarrow \infty}(x(t), y(t), u(t), v(t)) .
$$


Furthermore, let us define the three-dimensional solid $\Omega$ by

$$
\Omega \equiv \text { closure of } \bigcup_{t \geq 0}\{(x(t), y(t), u(t), v(t)):(x(0), y(0), u(0), v(0)) \in D\} .
$$

Observe that $\Omega$ can be equipped with an orientation determined by an orientation of $D$, since $D$ will be a face of $\Omega$.

If $\left(z_{0}, w_{0}\right)$ is the sole critical point accessible from the real planar domain $D$, $\left(z_{0}, w_{0}\right) \notin D$, then $\Omega$ will have a boundary comprised of three components: $D$, the union of trajectories of (4.1) issuing from $\partial D$, and a surface $\Sigma$ containing $\left(z_{0}, w_{0}\right)$. Let us call $\Sigma$ the steepest descent surface of $S$ through $\left(z_{0}, w_{0}\right)$ determined by $D$. Defined this way, $\Sigma$ is uniquely determined.

For the choice of phase function in (2.2), we can explicitly determine $\Sigma$.

Observe that if we set $x(0)=0$, and $v(0)=0$ as initial conditions in (4.1), then

$$
-\nabla \operatorname{Re} S(0, y, u, 0)=\left(0,\left(1-y^{2}\right)\left(1+u^{2}\right), 2 u y\left(1-y^{2} / 3\right), 0\right)
$$

whence we obtain $\dot{x}=\dot{v}=0$. In turn, this gives, for all $t \geq 0, x(t) \equiv v(t) \equiv$ 0 . Therefore, the gradient system (4.1) with initial data $(x(0), y(0), u(0), v(0))=$ $(0,0, u, 0) \in D$ results in a flow that is entirely contained in the $y u$-plane of $\mathbb{C} \times \mathbb{C}$. (Other trajectories, of course, do not lie within planes.)

This restricted system,

$$
\dot{y}=\left(1+u^{2}\right)\left(1-y^{2}\right), \quad \dot{u}=2 u y\left(1-y^{2} / 3\right),
$$

gives rise to the trajectories presented in Fig. 2. Observe that only the critical point

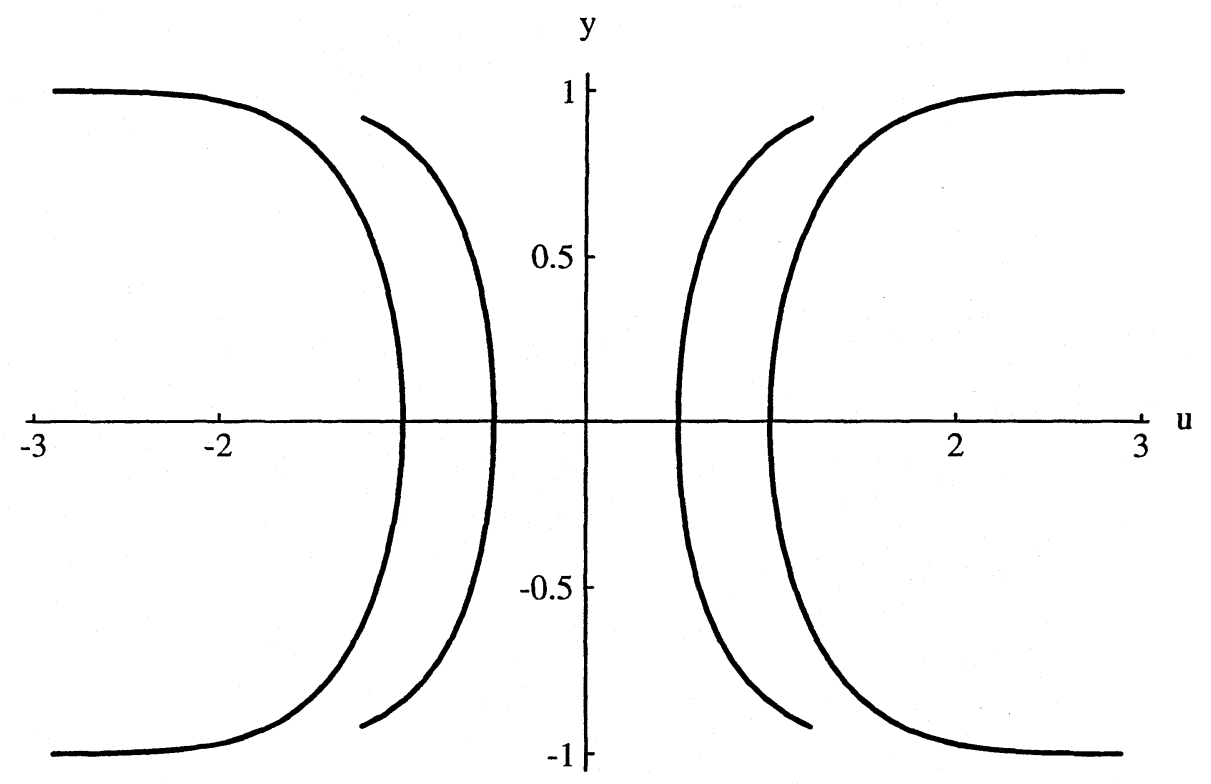

Figure 2. Flows of the restricted gradient system (3.4).

$(u, y)=(0,1)$ is accessible by a trajectory from the $u$-axis. Thus, since this is the $x=v=0$ slice of $\mathbb{C} \times \mathbb{C}$, we see that the saddle point $\left(z_{0}, w_{0}\right)=(i, 0)$ is accessible from the domain $D$. 
We also see that the steepest descent surface $\Sigma$ contains the line $u \mapsto(i, u) \subset \mathbb{C} \times \mathbb{C}$, with $u$ ranging over the real numbers.

More, in fact, can be said. Let us say that a surface $\mathcal{S}$ is obtained through coördinatewise steepest descent if $\mathcal{S}$ contains a saddle point $\left(z_{0}, w_{0}\right)$ of a holomorphic function, $f(z, w)$, and $\mathcal{S}$ is obtained by first constructing the steepest descent curve $\operatorname{Im} f\left(z, w_{0}\right)=\operatorname{Im} f\left(z_{0}, w_{0}\right)$, and then, at each point $\left(z^{\prime}, w_{0}\right)$ along this section, one fashions the steepest descent curves $\operatorname{Im} f\left(z^{\prime}, w\right)=\operatorname{Im} f\left(z^{\prime}, w_{0}\right)\left(=\operatorname{Im} f\left(z_{0}, w_{0}\right)\right)$.

Proposition 4.1. Let $f(z, w)$ be an entire function, with saddle point $\left(z_{0}, w_{0}\right)$ not contained in a domain $D$. If $\mathcal{S}$ is the steepest descent surface for $f$ through $\left(z_{0}, w_{0}\right)$, and if $\left(z_{0}, w_{0}\right)$ is accessible from $D$, then $\mathcal{S}$ is a coördinatewise steepest descent surface.

Proof. This is a simple consequence of the alternative form of the autonomous system (4.1):

$$
(\dot{z}, \dot{w})=-\left(\overline{f_{z}}, \overline{f_{w}}\right)
$$

where the overline indicates complex conjugation. Let $\left(z^{\prime}, w^{\prime}\right)$ be a point of $\mathcal{S}$. The steepest descent curve through $z=z^{\prime}$ of $f\left(z, w^{\prime}\right)$ is obtained from $\dot{z}=-\overline{f_{z}\left(z, w^{\prime}\right)}$, and . the steepest descent curve through $w=w^{\prime}$ of $f\left(z^{\prime}, w\right)$ comes from $\dot{w}=-\overline{f_{w}\left(z^{\prime}, w\right)}$. Both of these cases, at $\left(z^{\prime}, w^{\prime}\right)$, are subsumed in (4.5).

The restriction of accessibility from a domain can be removed, since the domain $D$ was arbitrary.

Returning to our phase function $S$ and our steepest descent surface $\Sigma$, we see that an explicit characterisation of $\Sigma$ can be obtained by taking a point of the line we know $\Sigma$ contains, and then constructing steepest descent curves through points of that line.

At the critical point $\left(z_{0}, w_{0}\right)=(i, 0)$, if we look for steepest descent curves in the $w=0$ section, we obtain one arch of the hyperbola $y^{2}=x^{2} / 3+1$ (the branch that, in the $z=x+i y$ plane, passes through $(x, y)=(0,1))$. Repeating the analysis for $z$-plane steepest descent curves through $(z, w)=(i, u), u$ real, results in the same curve, as can be seen from

$$
\operatorname{Im} S(x, y, u, v)=x\left(1+u^{2}\right)\left(1+x^{2} / 3-y^{2}\right)=\operatorname{Im} S(0,1, u, 0)=0 .
$$

Consequently, $\Sigma$ is nothing more than a Cartesian product of a line in the plane with one branch of a hyperbola.

\section{The contribution of the saddle point}

Let us define $I_{\Sigma}(\lambda)$ by

$$
I_{\Sigma}(\lambda)=\iint_{\Sigma} e^{\lambda S(z, w)} d z d w
$$

If we can parametrize $\Sigma$, we can easily develop an asymptotic expansion of $I_{\Sigma}$ for large $\lambda$.

We seek a parametrisation $z=z(\sigma, \tau), w=w(\sigma, \tau)$ such that

$$
(z, w):(\sigma, \tau) \mapsto \Sigma, \quad \sigma, \tau \text { real, }
$$

and satisfying $(z(0,0), w(0,0))=(i, 0)$. Ideally, the phase $S$, when evaluated at $(z(\sigma, \tau), w(\sigma, \tau))$, should result in an expression of the form const $-\sigma^{2}-\tau^{2}$, paralleling in a natural way what happens for steepest descent in the complex plane. The 
sum of squares could readily be replaced by any nonnegative real-valued function of $\sigma$ and $\tau$ that vanishes only at its solitary critical point $\sigma=\tau=0$.

Since $\Sigma$ is a Cartesian product, we can find a parametrisation of the form $z=z(\sigma)$, $w=w(\tau)$. Furthermore, since $\Sigma$ is a ruled surface, with the rulings occuring along the Re $w$-direction, a natural choice for $w(\tau)$ is $w(\tau)=\tau$. With this choice for $w$, we find that

$$
\begin{aligned}
\operatorname{Im}[S(z(\sigma), w(\tau))-S(i, 0)] & =\operatorname{Im}\left[i\left(\tau^{2}+1\right)\left(z(\sigma)^{3} / 3+z(\sigma)-2 i / 3\right)\right] \\
& =\left(\tau^{2}+1\right)\left(x(\sigma)^{3} / 3-x(\sigma) y(\sigma)^{2}+x(\sigma)\right),
\end{aligned}
$$

where, as before, $z=x+i y$. Since this expression must vanish, we must have $x=0$ or $x^{2} / 3-y^{2}+1=0$. The choice

$$
x(\sigma)=\sqrt{3} \sinh \sigma, \quad y(\sigma)=\cosh \sigma
$$

is evidently a parametrisation which will satisfy $z(0)=i$. A parametrisation of $\Sigma$ is therefore furnished by

$$
(z, w)=(\sqrt{3} \sinh \sigma+i \cosh \sigma, \tau) .
$$

It is readily verified that $S(z(\sigma), w(\tau))$ has its only critical point at the origin, and that the origin gives a global maximum. Furthermore,

$$
\begin{aligned}
S(z(\sigma), w(\tau))-S(i, 0) & =\frac{2}{3}\left[1-\left(1+\tau^{2}\right)\left(1+4 \sinh ^{2} \sigma\right) \cosh \sigma\right] \\
& =-3 \sigma^{2}-\frac{2}{3} \tau^{2}-\frac{9}{4} \sigma^{4}-3 \sigma^{2} \tau^{2}+\mathcal{O}(5), \quad(\sigma, \tau \rightarrow 0),
\end{aligned}
$$

where the big 'oh' in the second line indicates terms of order $\geq 5$.

Thus, we may write

$$
\begin{aligned}
I_{\Sigma}(\lambda) & =\iint_{\Sigma} e^{\lambda S} d z d w=e^{\lambda S(i, 0)} \iint_{\Sigma} e^{\lambda[S(z, w)-S(i, 0)]} d z d w \\
& =e^{-2 \lambda / 3} \int_{-\infty}^{+\infty} \int_{-\infty}^{+\infty} e^{\frac{2}{3} \lambda\left[1-\left(1+\tau^{2}\right)\left(1+4 \sinh ^{2} \sigma\right) \cosh \sigma\right]}(\sqrt{3} \cosh \sigma+i \sinh \sigma) d \sigma d \tau
\end{aligned}
$$

upon making use of the parametrisation (5.2). In view of (5.3), we transform to $u \tau$-coördinates by setting

$$
\begin{aligned}
u^{2} & =\frac{2}{9}\left(1+\tau^{2}\right)\left(\left(1+4 \sinh ^{2} \sigma\right) \cosh \sigma-1\right) \\
& =\sigma^{2}\left(1+\tau^{2}+\frac{3}{4} \sigma^{2}+\cdots\right) \quad(\sigma, \tau \rightarrow 0)
\end{aligned}
$$

from which we see that $u=\sigma+$ higher order terms near the origin. Accordingly, our integral becomes

$$
\begin{aligned}
I_{\Sigma}(\lambda) & =e^{-2 \lambda / 3} \int_{-\infty}^{+\infty} \int_{-\infty}^{+\infty} e^{-\lambda\left(3 u^{2}+\frac{2}{3} \tau^{2}\right)}(\sqrt{3} \cosh \sigma(u, \tau)+i \sinh \sigma(u, \tau)) \frac{\partial(\sigma, \tau)}{\partial(u, \tau)} d u d \tau \\
& =\sqrt{3} e^{-2 \lambda / 3} \int_{-\infty}^{+\infty} \int_{-\infty}^{+\infty} e^{-\lambda\left(3 u^{2}+\frac{2}{3} \tau^{2}\right)} G(u, \tau) d u d \tau
\end{aligned}
$$


where we have set

$$
G(u, \tau)=\left(\cosh \sigma(u, \tau)+\frac{i}{\sqrt{3}} \sinh \sigma(u, \tau)\right) \frac{\partial(\sigma, \tau)}{\partial(u, \tau)} \equiv \sum_{k, l=0}^{\infty} g_{k l} u^{k} \tau^{l}
$$

Finally, the change to polar coördinates $u=(1 / \sqrt{3}) r \cos \theta, \tau=\sqrt{\frac{3}{2}} r \sin \theta$ produces

$$
I_{\Sigma}(\lambda) \sim \sqrt{\frac{3}{2}} e^{-2 \lambda / 3} \sum_{n=0}^{\infty} \sum_{k+l=n} g_{k l} \frac{3^{(l-k) / 2}}{2^{l / 2}} \int_{0}^{\infty} e^{-\lambda r^{2}} r^{k+l+1} d r \int_{0}^{2 \pi} \cos ^{k} \theta \sin ^{l} \theta d \theta
$$

In view of the evaluation $2 \int_{0}^{\pi / 2} \cos ^{\mu-1} x \sin ^{\nu-1} x d x=B(\mu / 2, \nu / 2)$, where $B$ is the beta function, we may write, instead,

$$
I_{\Sigma}(\lambda) \sim \sqrt{\frac{3}{2}} \frac{e^{-2 \lambda / 3}}{\lambda} \sum_{n=0}^{\infty} \sum_{k+l=n} \frac{3^{(l-k) / 2}}{2^{l / 2}} g_{k l} c_{k l} \frac{\Gamma((k+1) / 2) \Gamma((l+1) / 2)}{\lambda^{(k+l) / 2}}
$$

where the numbers $c_{k l}$ are zero if either $k$ or $l$ is odd, and 1 for $k$ and $l$ both even. This reduces further to

$$
I_{\Sigma}(\lambda) \sim \sqrt{\frac{3}{2}} \frac{e^{-2 \lambda / 3}}{\lambda} \sum_{n=0}^{\infty} \frac{1}{\lambda^{n}} \sum_{k=0}^{n} \frac{3^{n-2 k}}{2^{n-k}} g_{2 k, 2 n-2 k} \Gamma(k+(1 / 2)) \Gamma(l+(1 / 2)),
$$

whence use of the relation

$$
\Gamma(s+(1 / 2))=(1 / 2)_{s} \Gamma(1 / 2)
$$

results in

$$
I_{\Sigma}(\lambda) \sim \frac{\pi}{\lambda} \sqrt{\frac{3}{2}} e^{-2 \lambda / 3} \sum_{n=0}^{\infty}\left(\frac{3}{2}\right)^{n} \frac{1}{\lambda^{n}} \sum_{k=0}^{n}\left(\frac{2}{9}\right)^{k} g_{2 k, 2 n-2 k}\left(\frac{1}{2}\right)_{k}\left(\frac{1}{2}\right)_{n-k} .
$$

The numbers $g_{i j}$ are readily determined. The first few values are

$$
\begin{aligned}
& g_{00}=1 \\
& g_{10}=i / \sqrt{3}, \quad g_{01}=0, \\
& g_{20}=-5 / 8, \quad g_{11}=0, \quad g_{02}=-1 / 2 \text {, } \\
& g_{30}=-4 i /(3 \sqrt{3}), \quad g_{21}=0, \quad g_{12}=-i / \sqrt{3}, \quad g_{03}=0 \text {. }
\end{aligned}
$$

An approximation to $I_{\Sigma}$, for subsequent use, is therefore

$$
I_{\Sigma}(\lambda)=\frac{\pi}{\lambda} \sqrt{\frac{3}{2}} e^{-2 \lambda / 3}\left[1-\frac{7}{24 \lambda}+\mathcal{O}\left(\frac{1}{\lambda^{2}}\right)\right]
$$

as $\lambda \rightarrow+\infty$. 


\section{Deforming the integration domain $D$}

Applying the deformation process (4.1) results in five smooth pieces: four pieces result from the integral curves issuing from each edge of $D$; the final piece is $\Sigma$.

Call the pieces resulting from the edges $F_{1}, \ldots, F_{4}$. By their construction, $e^{\lambda S(z, w)}$ decays exponentially along each $F_{i}$. Observe, too, that although the surface obtained by forming $\bigcup F_{i}$ is non-smooth along trajectories issuing from the corners of $D$, when $\Omega$ is equipped with an orientation, the induced orientation on the $F_{i}$ 's is such that the contributions to the asymptotic behaviour of the integral taken over each "face" cancel in pairs along the trajectories issuing from the corners of $D$.

It is also clear that $D$ and $\Sigma$ must be oppositely oriented. This follows from consideration of the orientation of $\Omega$ induced on the pieces $F_{1}, \ldots, F_{4}, \Sigma$ and $D$; see Fig. 3 .

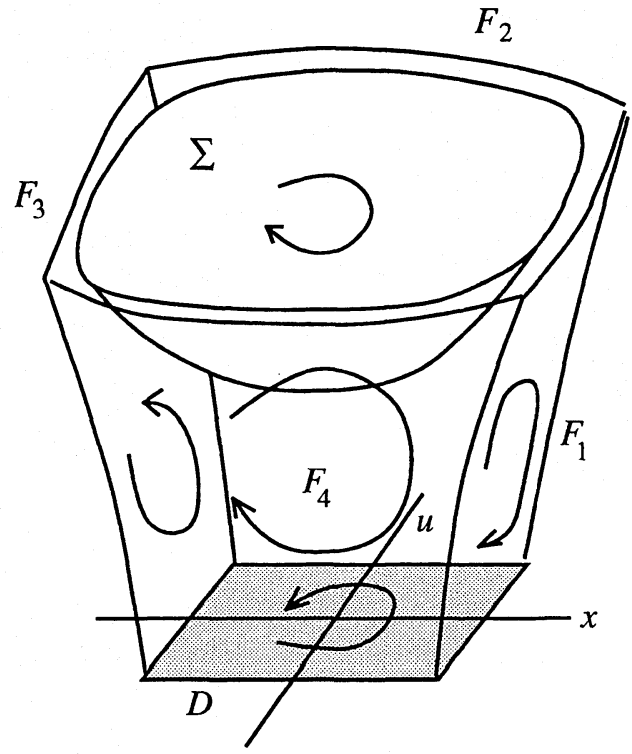

FIGURE 3. Orientation of the boundary of $\Omega$.

Accordingly, with the orientations depicted in Fig. 3, we have

$$
I_{\partial \Omega}(\lambda)=I_{D}(\lambda)+I_{-\Sigma}(\lambda)+\sum_{i=1}^{4} I_{F_{i}}(\lambda) \equiv 0
$$

or

$$
I(\lambda) \equiv I_{D}(\lambda)=I_{\Sigma}(\lambda)-\sum_{i=1}^{4} I_{F_{i}}(\lambda),
$$

where $I_{U}$ indicates the integral $\iint_{U} e^{\lambda S} d z d w$. The contributions of the "face" integrals amount to nothing other than the contributions to the value of $I_{D}$ made by boundary stationary points and corner points of the domain $D$. Routine computation reveals that the corner points of $D$ give rise to the approximation

$$
-\frac{3}{8 \lambda^{2}} \cos \left(\frac{8 \lambda}{3}\right)+\mathcal{O}\left(\frac{1}{\lambda^{3}}\right)
$$


while the boundary stationary points at $(z, w)=(1,0)$ and $(z, w)=(0,-1)$ give rise to the approximation

$$
\frac{\sqrt{3 \pi}}{2 \lambda^{3 / 2}} \cos \left(\frac{4 \lambda}{3}-\frac{\pi}{4}\right)+\mathcal{O}\left(\frac{1}{\lambda^{5 / 2}}\right) .
$$

Combining these two approximations gives rise to the usual stationary phase approximation

$$
I_{D}(\lambda) \sim A_{U}(\lambda) \equiv \frac{\sqrt{3 \pi}}{2 \lambda^{3 / 2}} \cos \left(\frac{4 \lambda}{3}-\frac{\pi}{4}\right)-\frac{3}{8 \lambda^{2}} \cos \left(\frac{8 \lambda}{3}\right) .
$$

However, in view of (6.1) and (5.6), we have the improved approximation

$$
I_{D}(\lambda) \sim A_{U}(\lambda)+\frac{\pi}{\lambda} \sqrt{\frac{3}{2}} e^{-2 \lambda / 3}\left[1-\frac{7}{24 \lambda}\right] \equiv A_{E}(\lambda) .
$$

\section{Closing remarks}

Some idea of the improvement obtained by including the exponentially decaying term $I_{\Sigma}$ can be seen by examining the approximations (6.2) and (6.3) and comparing the errors committed by each approximation. To that end, we computed several values of $I(\lambda)$ for a range of small values of $\lambda$ by representing $I(\lambda)$ as a power series. The result of these computations is presented in the accompanying table.

\begin{tabular}{c||c|cc||c|c}
\multicolumn{1}{c}{$\lambda$} & $I(\lambda)$ & $A_{U}(\lambda)$ & \multicolumn{1}{c}{$A_{E}(\lambda)$} & \multicolumn{1}{c}{$A_{U}(\lambda)-I(\lambda)$} & $A_{E}(\lambda)-I(\lambda)$ \\
\hline 3.2 & -0.112357 & -0.254251 & -0.124818 & -0.1419 & -0.0125 \\
3.4 & -0.0863760 & -0.206723 & -0.0994809 & -0.1203 & -0.0131 \\
3.6 & -0.0554468 & -0.157753 & -0.0686500 & -0.1023 & -0.0132 \\
3.8 & -0.0237642 & -0.110225 & -0.0360057 & -0.0865 & -0.0122 \\
4.0 & 0.00673929 & -0.0652479 & -0.00328443 & -0.0720 & -0.0100 \\
\hline 4.2 & 0.0354874 & -0.0230782 & 0.0287617 & -0.0586 & -0.0067 \\
4.4 & 0.0623603 & 0.0160900 & 0.0595436 & -0.0463 & -0.0028 \\
4.6 & 0.0870081 & 0.0516095 & 0.0880977 & -0.0354 & 0.0011 \\
4.8 & 0.108499 & 0.0822101 & 0.112899 & -0.0263 & 0.0044 \\
5.0 & 0.125328 & 0.106154 & 0.132005 & -0.0192 & 0.0067 \\
\hline 5.2 & 0.135713 & 0.121624 & 0.143430 & -0.0141 & 0.0077 \\
5.4 & 0.138062 & 0.127209 & 0.145626 & -0.0109 & 0.0076 \\
5.6 & 0.131444 & 0.122335 & 0.137910 & -0.0091 & 0.0065 \\
5.8 & 0.115936 & 0.107543 & 0.120729 & -0.0084 & 0.0048 \\
6.0 & 0.0927487 & 0.0845190 & 0.0956934 & -0.0082 & 0.0029 \\
\hline
\end{tabular}

TABLE 1. Comparison of absolute errors committed by the usual stationary phase method, and the stationary phase method augmented with the inclusion of exponentially small terms.

As is apparent, the absolute errors committed by either approximation decrease as $\lambda$ increases, but the improved approximation commits an absolute error that is consistently smaller than that committed by the usual result of the method of stationary phase. 
Before closing, we mention the work of Ursell in [Urs], in which integrals of the form

$$
\iint_{S} G(x, y, \alpha, \beta, \gamma) \exp \left\{i N\left(x^{3} / 3+y^{3} / 3+\alpha x+\beta y+\gamma x y\right)\right\} d x d y
$$

are considered. Here, $S$ is either a bounded surface, or an unbounded surface over which the integrand exhibits exponential decay for large $|x|+|y|$. The parameters $\alpha$, $\beta$ and $\gamma$ are small complex numbers, and the goal of the work in [Urs] is to develop a large $N$ asymptotic expansion of (7.1) which remains uniformly valid for all sufficiently small $|\alpha|,|\beta|$ and $|\gamma|$.

By working with a perturbation of the phase function of (7.1) (effectively, replacing $\alpha$ by $\alpha+\sigma$ and $\beta$ with $\beta+\tau$ ), and taking advantage of the fact that the surface of steepest descent for the case $\gamma=0$ is a Cartesian product of two plane curves (one in the $x$-plane, one in the $y$-plane), Professor Ursell is able to construct a suitable surface over which the saddle point method can be applied. The constructions he uses (especially in $\S 4$ of [Urs]) resemble in many respects the idea of coördinatewise steepest descent surfaces. An important difference, however, is the systematic deformation process we used in order to determine which surfaces through complex stationary points were to be used in the development of the asymptotics of (2.1).

There remain many points to be addressed with the technique described in this paper, some of which concern $\Omega$ and the holomorphicity of $f$ and $g$; recall eqn. (1.1). For example, it is unknown what expansion results when the deformation process creates an $\Omega$ in which part of $\Omega$ lies outside the domain of holomorphy of $g$. A similar question can be raised in the circumstance when $\Sigma$, or one of the "faces" $F_{i}$, is tangent to the domain of holomorphy of $g$.

A more pressing concern is the representation of (2.1) in the form (6.1). The discussion in $\S 6$ is of an intuitive character, and lacks rigour. This is due, in part, to the fact that the trajectories that constitute $\Omega$ can only be studied qualitatively. A more appealing way of obtaining (6.1) would be to proceed by considering a ball of radius $N$, say,

$$
B_{N}=\left\{(z, w):|z|^{2}+|w|^{2} \leq N\right\}
$$

and forming

$$
\Omega_{N} \equiv \Omega \cap B_{N}
$$

$\Omega_{N}$ would have a boundary consisting of portions of the faces $F_{i}$, a portion of $\Sigma$, and a new piece, say $S_{N}$, contained in $\partial B_{N}$. Since the vector field $\nabla \operatorname{Re} S$ has only finitely many critical points, for large enough $N, \nabla \operatorname{Re} S$ is not tangent to $\partial B_{N}$. From this, we can conclude $S_{N}$ is of dimension 2 .

To show that a relationship such as (6.1) holds, it would suffice to show that

$$
\int_{S_{N}} e^{\lambda S} d z d w=o(1) \text { as } N \rightarrow \infty
$$

Since $e^{\lambda S}$ decays exponentially on $\Omega$ as $|z|+|w| \rightarrow . \infty,(7.2)$ follows if it can be shown that $\operatorname{Vol}\left(S_{N}\right)=\mathcal{O}\left(N^{\epsilon}\right)$ for some real $\epsilon$. This seems intuitively clear, but computing $S_{N}$ from the trajectories issuing from $D$ is virtually impossible in all but the most simple cases. Success in this approach may come from a study of the large-scale structure of $\Sigma$ and the $F_{i}$ 's. Some preliminary results of such a study can be found in [Kam2]. 


\section{References}

[BeH] M. V. Berry, and C. J. Howls, Hyperasymptotics for integrals with saddles, Proc. R. Soc. Lond., Series A 434 (1991), 657-675.

[BH] N. Bleistein, and R. A. Handelsman, Asymptotic Expansions of Integrals, Holt, Rinehart \& Winston, New York, 1975.

[Fed] M. V. Fedoryuk, Metod Perevala (in Russian), Nauka, Moscow, 1977.

[IN] H. Ikuno, and M. Nishimoto, Calculation of transfer functions of three-dimensional indented objects by the physical optics approximation combined with the method of stationary phase, IEEE Transactions on Antennas and Propagation 39 (1991), 585-590.

[Jones] D. S. Jones, Generalised Functions, McGraw-Hill, New York, 1966.

[Kam1] D. Kaminski, On the n-variable saddle point and steepest descent methods, in Asymptotic and Computational Analysis, Lecture Notes in Pure and Applied Math. Series, No. 124, R. Wong, ed., Marcel-Dekker, New York (1990), 627-637.

[Kam2] _ Steepest descent curves via vector fields, Technical report TR-MA-01-91, University of Lethbridge, 1991.

[Olv] F. W. J. Olver, Asymptotics and Special Functions, Academic Press, Inc., New York, 1974.

[Pham] F. Pham, Vanishing homologies and the $n$-variable saddlepoint method, Proc. Symposia in Pure Math. 40 (1983), 319-33.

[Urs] F. Ursell, Integrals with a large parameter: a double complex integral with four nearly coincident saddle-points, Math. Proc. Camb. Phil. Soc. 87 (1980), 249-273.

[Wong] R. Wong, Asymptotic Approximations of Integrals, Academic Press, Inc., Toronto, 1989.

Department of Mathematics and Computer Science, University of Lethbridge, 4401 University Drive, Lethbridge, Alberta, Canada T1K 3M4

E-mail address: kaminski@cs.uleth.ca 